\title{
Perfil da pesquisa acadêmica odontológica da EBMSP
}

Renato Magalhães Costa*; Raquel da Silva Vieira**; Andréa Nóbrega Cavalcanti ${ }^{* * *}$; Urbino da Rocha Tunes $^{* * * *}$; Érica Del Peloso Ribeiro ${ }^{* * * * *}$; Viviane Maia Barreto de Oliveira ${ }^{* * * * * *}$

* Cirurgião-dentista pela Escola Bahiana de Medicina e Saúde Pública (EBSMP)

** Cirurgiã-dentista pela Escola Bahiana de Medicina e Saúde Pública (EBSMP)

**** Doutora em Clínica Odontológica (Dentística) pela UNICAMP e Professora Adjunta da Escola Bahiana de Medicina e Saúde Pública (EBSMP)

**** Coordenador do Curso de Graduação em Odontologia e Professor Titular de Periodontia da Escola Bahiana de Medicina e Saúde Pública (EBSMP)

***** Doutora em Clínica Odontológica (Periodontia) pela UNICAMP e Professora Adjunta do Componente Curricular TCC2, TCC 3 e TCC Defesa da Escola Bahiana de Medicina e Saúde Pública (EBMSP)

****** Doutora em Clínica Odontológica (Prótese) pela UNICAMP e Professora Adjunta do Componente Curricular TCC1, TCC 3 e TCC Defesa da Escola Bahiana de Medicina e Saúde Pública (EBMSP)

\section{RESUMO}

As Diretrizes Curriculares Nacionais do Curso de Odontologia (DCN), Resolução CNE/CES 03 de 2002, no artigo 12, instituem que, para conclusão do Curso de Graduação em Odontologia, o aluno deverá elaborar um trabalho sob orientação docente. No Curso de Odontologia da Escola Bahiana de Medicina e Saúde Pública (EBMSP), o Trabalho de Conclusão de Curso (TCC) deve ser redigido na forma de um artigo científico, sendo possível produzir uma pesquisa, revisão de literatura ou relato de caso. O objetivo deste estudo foi avaliar o perfil de pesquisa acadêmica do curso de Odontologia da EBMSP através da análise de todos TCCs apresentados no período de 2007/2 a 2012/1. Os trabalhos foram classificados em pesquisa, revisão de literatura ou relato de caso e em relação à área. Dos 314 TCCs, 114 (36,3\%) foram pesquisas, 109 (34,7\%) relatos de caso e 91 (29\%) revisões. Somente 21 (6,7\%) trabalhos apresentaram um coorientador. Prótese, estomatologia, cirurgia e traumatologia bucomaxilofacial (CTBMF), periodontia e dentís- tica foram as áreas mais escolhidas. Os trabalhos foram orientados ou coorientados por docente com doutorado em 59,2\% (186) dos casos. Dos trabalhos de pesquisa, 88 (72\%) tiveram orientação de um doutor, mas este número reduziu para 54 (49,5\%) dos relatos de caso e 45 (49,5\%) das revisões. Concluise que prótese, estomatologia e CTBMF estiveram entre as áreas mais selecionadas, tanto em uma avaliação global, quanto quando subdividas por tipos de trabalho. A titulação de doutorado do orientador mostrou-se um relevante fator na produção de pesquisas.

Descritores: Pesquisa em Odontologia. Ensino. Bibliometria.

\section{INTRODUÇÃO}

A forma como o saber é construído e divulgado é de suma importância, uma vez que influenciará e norteará os pensamentos, as reflexões e as atitudes, moldando os fazeres em todos os campos do conhecimento ${ }^{1}$. 
A resolução CNE/CES 3, de 19 de fevereiro de 2002, apresenta as Diretrizes Curriculares Nacionais do Curso de Graduação em Odontologia ${ }^{2}$. As Diretrizes são fruto da participação de diferentes instituições, organizações e especialistas da sociedade civil organizada e também do Estado, com ampla consulta em audiências e eventos públicos ${ }^{3}$.

O artigo 12 da resolução institui: "Para conclusão do Curso de Graduação em Odontologia, o aluno deverá elaborar um trabalho sob orientação docente", fazendo uma clara referência ao Trabalho de Conclusão de Curso (TCC). Entretanto, nenhuma informação adicional é apresentada, como por exemplo, o formato do trabalho e quando se deve iniciar a sua confecção.

Compreender o que tem motivado os alunos na seleção dos temas de TCC e correlacionar a formação do professor no tipo de trabalho executado na graduação é de fundamental importância para delinear os novos rumos da pesquisa científica no meio acadêmico.

Dessa forma, o objetivo deste estudo é avaliar o perfil de pesquisa acadêmica do Curso de Odontologia da EBMSP através da análise de todos TCCs apresentados no período de 2007/2 a 2012/1.

\section{METODOLOGIA}

Este é um estudo retrospectivo, longitudinal, com caráter exploratório e abordagem quantitativa. Foram avaliados os resumos de todos os TCCs defendidos no período 2007/2 a 2012/1, no Curso de Odontologia da Escola Bahiana de Medicina e Saúde Pública (EBMSP), o que resultou em uma amostra de 314 trabalhos. Esses trabalhos foram subdivididos em relação à área de concentração e ao tipo de estudo (pesquisa, relato de caso ou revisão de literatura) e foram tabulados em valores percentuais. Também foi realizada uma análise correlacional da titulação do professor e o tipo de estudo.

\section{RESULTADOS}

Dos 314 TCC's, 114 (36,3\%) foram pesquisas, 109 (34,7\%) relatos de caso e 91 (29\%) revisões. Somente 21 (6,7\%) trabalhos apresentaram um coorientador. O Gráfico 1 traz a subdivisão dos trabalhos em relação à área do estudo.

Gráfico 1 - Trabalhos classificados em relação à área dos TCC’s

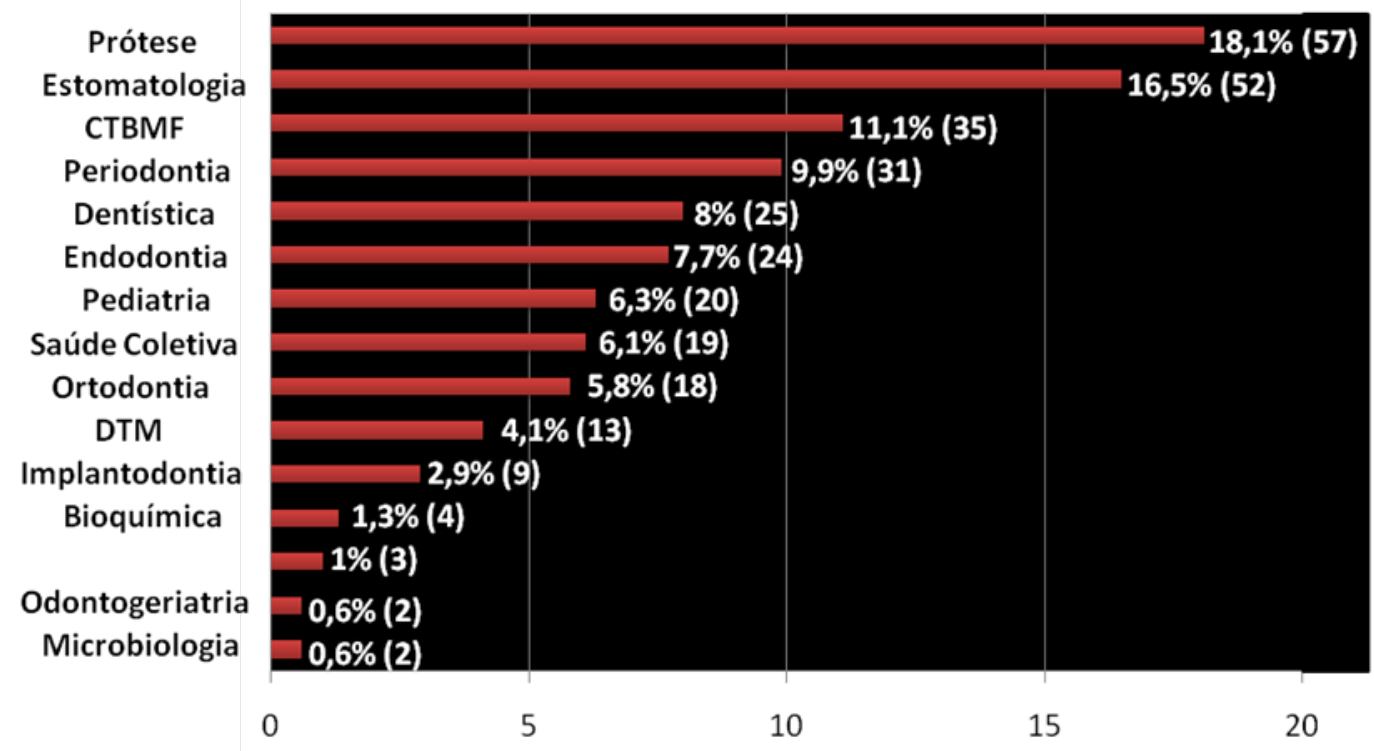


O Gráfico 2 mostra a contribuição de relato de caso, e o Gráfico 4 em relação a cada área nos trabalhos de pesquisa, o Gráfi- revisões de literatura.

co 3 apresenta essa relação nos trabalhos de

Gráfico 2 - Contribuição de cada área nos trabalhos de pesquisa

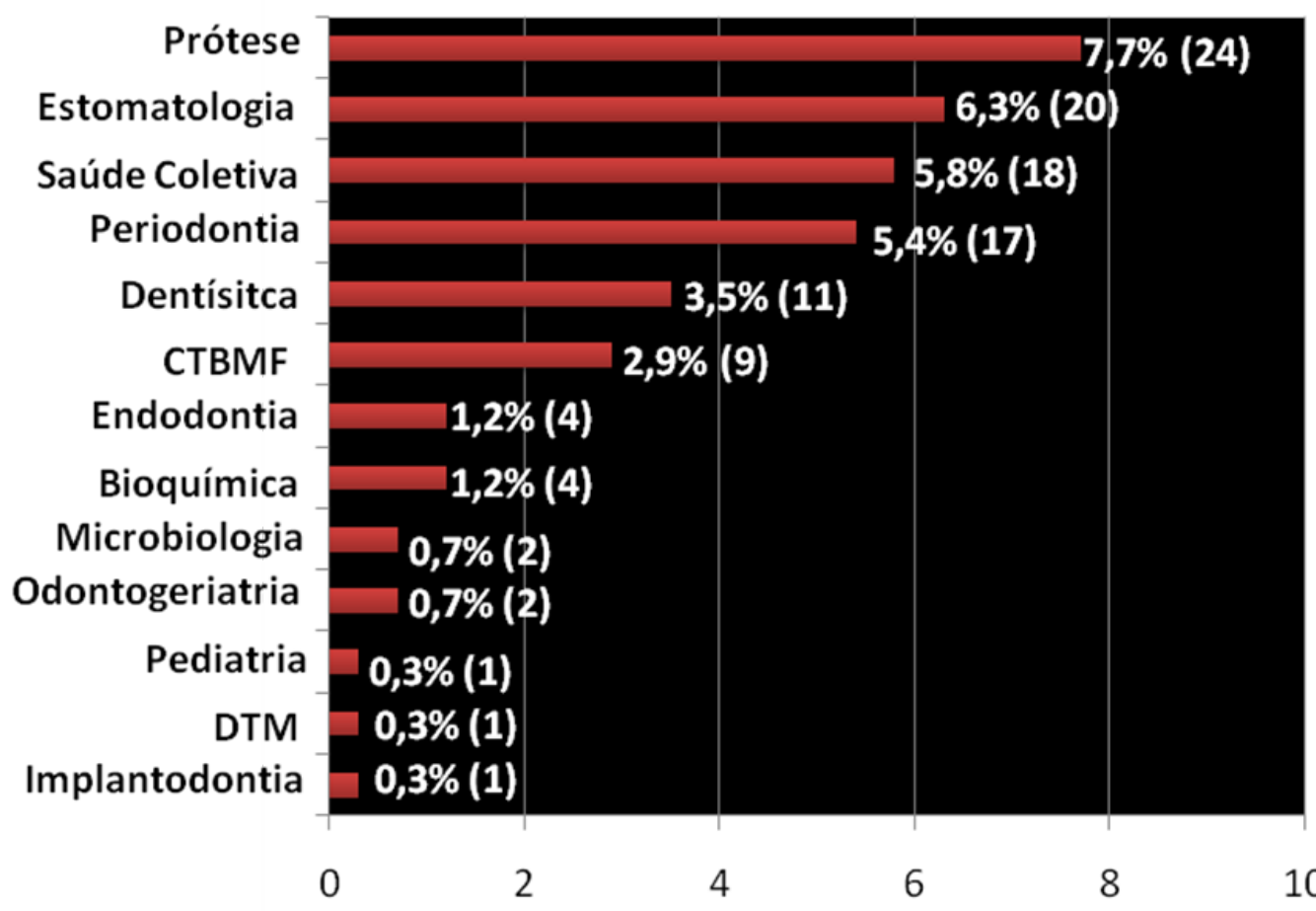

Gráfico 3 - Contribuição de cada área nos trabalhos de relato de caso

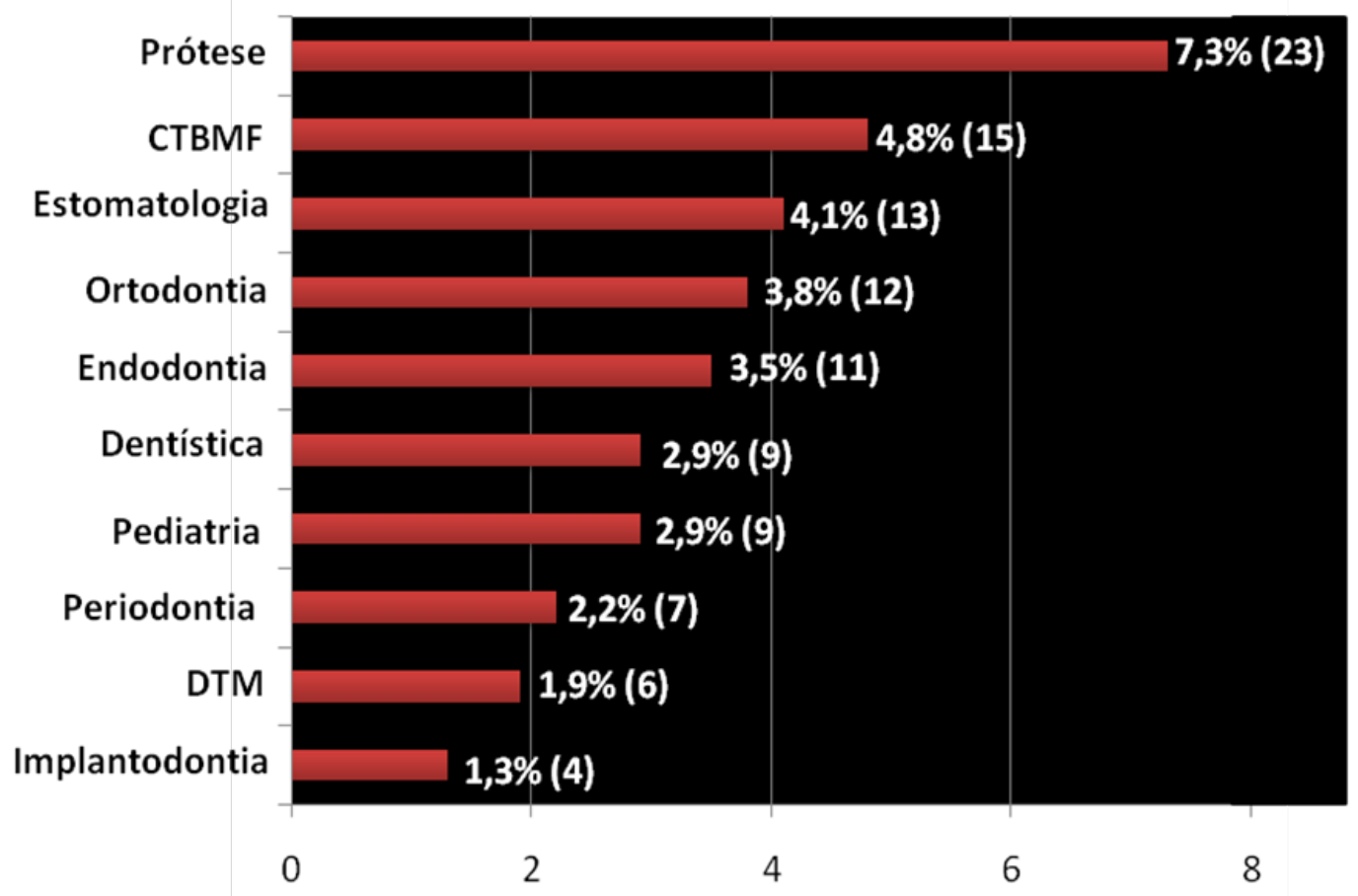


Gráfico 4 - Contribuição de cada área nos trabalhos de revisão de literatura

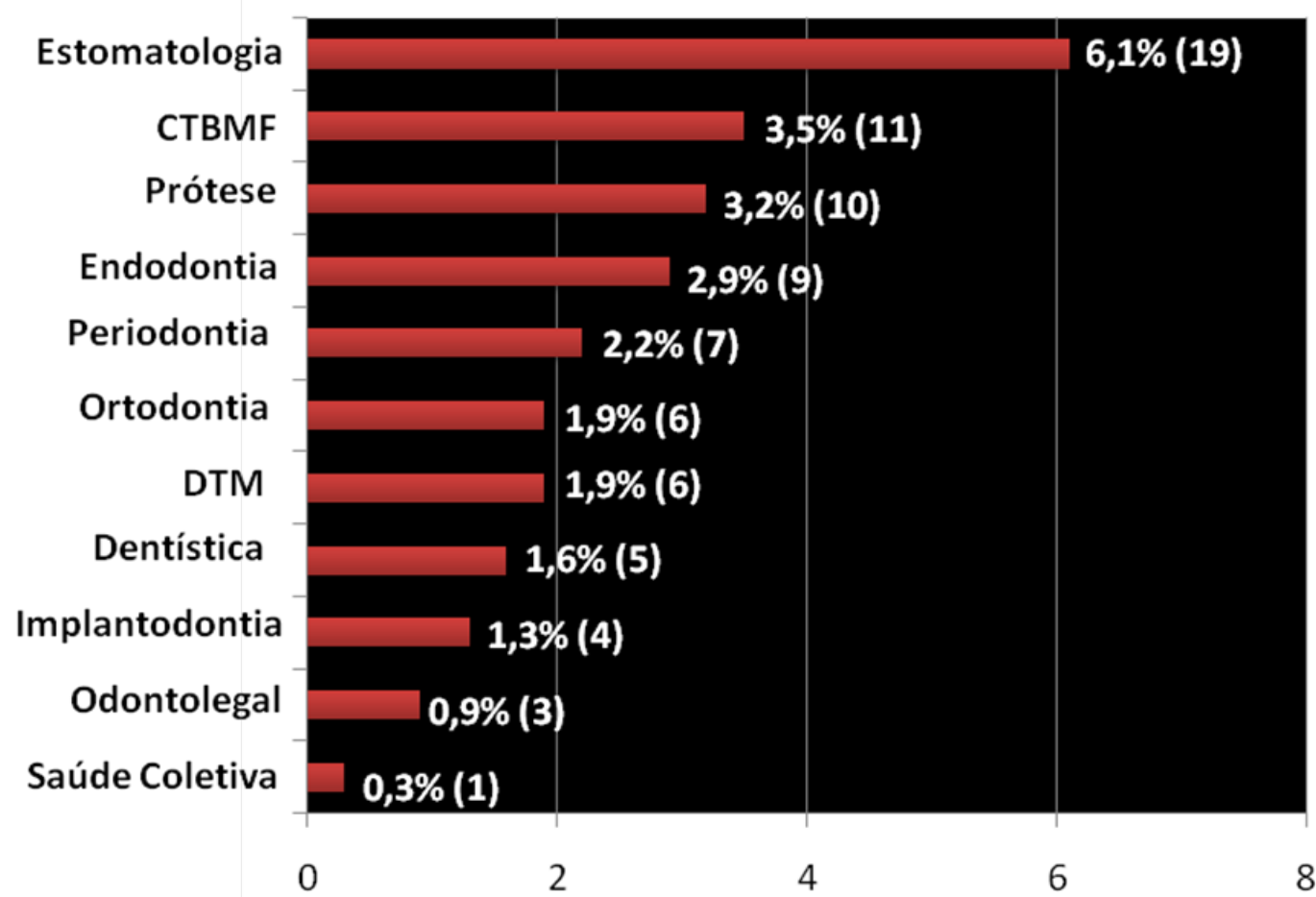

Em relação à titulação dos orientadores, 186 (59,2\%) trabalhos foram orientados e/ou coorientados por profissionais que possuíam doutorado no momento da defesa do TCC. Os trabalhos restantes, 128 (40,8\%), foram orientados por especialistas, mestres ou doutorandos.

Quando avaliados apenas trabalhos do tipo pesquisa, 88 (77,2\%) TCCs foram orientados por doutores e 26 (22,8\%) foram orientados por não doutores. Nas revisões de literatura, 45 (49,5\%) foram orientados por doutores e 46 (50,5\%) orientados por não doutores. Nos relatos de caso, a relação foi, respectivamente, 54 (49,5\%) e 55 (50,5\%).

\section{DISCUSSÃO}

Apesar de as DCN dos Cursos de Graduação em Odontologia preconizarem a realização do Trabalho de Conclusão de Curso, isso não é uma obrigatoriedade estabelecida pelo Ministério da Educação e Cultura (MEC).

$\mathrm{O}_{\mathrm{MEC}}^{4}$ esclarece que as DCN de cada curso definem a obrigatoriedade ou não de elaboração de TCC. A instituição de ensino (IES) deverá regulamentar as normas e os procedimentos e dar conhecimento ao aluno. Esses procedimentos devem ser explicitados no regimento da IES, o qual se constitui em documento que inclui os direitos e deveres relativos à comunidade acadêmica, bem como no projeto pedagógico do respectivo curso de nível superior. Ambos os documentos devem ser disponibilizados pela Instituição de Ensino.

No Curso de Odontologia da EBMSP, o TCC compõe quatro componentes curriculares: TCC I ( $7^{\circ}$ semestre), TCC II ( $8^{\circ}$ semestre), TCC III ( $9^{\circ}$ semestre) e TCC Defesa $\left(10^{\circ}\right.$ semestre). Este deve ser redigido na forma de um artigo científico e o discente pode realizar uma pesquisa, um relato de caso ou uma revisão de literatura.

Ao adotar o formato de TCC em artigo científico, a EBMSP visa facilitar a disseminação do resultado da pesquisa em periódicos, evitando que o resultado do trabalho desenvolvido permaneça apenas na própria instituição. 
No mundo científico moderno, em que observamos um aumento no número de pesquisas, as revistas científicas desempenham um papel importante, pois são maneiras dinâmicas de divulgar o conhecimento produzido ${ }^{5}$.

Refletindo criticamente, observa-se que as revistas científicas na área da odontologia, bem como nas demais áreas do saber, não têm apenas o propósito de estabelecer um elo de comunicação entre pesquisadores, profissionais e estudantes de Odontologia. Elas servem de depositárias das concepções que vão plasmando, moldando e dando existência à odontologia nacional e à ciência ${ }^{1}$.

Em avaliação global em relação à área do trabalho, prótese, estomatologia e CTBMF foram as mais prevalentes. Cabe ressaltar que estomatologia reúne trabalhos orientados por profissionais de radiologia, farmacologia, semiologia, patologia, CTBMF, odontopediatria e estomatologia.

Quando avaliados somente os trabalhos de pesquisa, verifica-se uma alta prevalência de prótese e estomatologia novamente. Já saúde coletiva, oitavo lugar na avaliação global, foi a terceira mais prevalente nas pesquisas. Os trabalhos realizados nessa área chamam atenção por serem trabalhos impactantes e com baixo custo. Por muitas vezes não necessitam de um ambiente físico específico, instrumentais ou materiais onerosos, e são realizados com pouco investimento financeiro. São impactantes porque são feitos com amostras numerosas, que levam à validade interna e externa.

Observa-se uma inter-relação entre a graduação e a pós-graduação da instituição. As três áreas de maior prevalência nos TCCs são opções de pós-graduação da EBMSP. Existem dois cursos de extensão de prótese; mestrado profissionalizante em odontologia na área de concentração de Estomatologia; assim como especialização, residência e três cursos de extensão em

CTBMF.

Em um estudo retrospectivo, Cavalcanti et al. ${ }^{6}$ analisaram 1.905 estudos apresentados na 20 $0^{\text {a }}$ Reunião Anual da Sociedade Brasileira de Pesquisa Odontológica. Verificaram que região sudeste concentrou o maior número de trabalhos $(76,4 \%)$. A região nordeste representava $7,2 \%$ das pesquisas, sendo 1,9\% provenientes da Bahia. O que desde aquela época ressaltava a dificuldade de desenvolvimento de pesquisas no Nordeste, principalmente na obtenção de recursos financeiros para esse fim. Isso explica a grande quantidade de pesquisas que envolvem baixo custo nesse Estado.

A odontologia - enquanto ciência da saúde - lida com a vida e com o sofrimento do ser humano. No seu processo de trabalho, evidenciam-se questões sociais, humanas e éticas, a partir das situações bilaterais que se estabelecem individualmente entre profissionais e pacientes e, coletivamente, entre a prática profissional e a sociedade ${ }^{7}$.

Garbin et al. ${ }^{8}$ avaliaram número, ano de registro e distribuição geográfica dos Comitês de Ética em Pesquisa (CEPs) existentes nas faculdades de medicina e odontologia brasileiras. Observou-se que 143 das 165 faculdades de medicina e 142 das 192 de odontologia apresentam CEPs registrados junto à Comissão Nacional de Ética em Pesquisa (CONEP). No Nordeste, $80 \%$ das faculdades de medicina e $74,2 \%$ das faculdades de odontologia apresentam CEP. Para os autores, existe um percentual relevante e inadmissível de faculdades que não apresentam este comitê.

A EBMSP conta com seu próprio CEP que atende a todos os cursos da instituição. Algumas dificuldades ainda são apontadas pelos pesquisadores para a submissão de 
projetos, principalmente quanto à informatização através da Plataforma Brasil.

Verifica-se, entretanto, que ao mesmo tempo em que ocorreram esses saltos tecnológicos e científicos, o número de pesquisadores e de instituições dedicadas ao ensino e à pesquisa aumentou consideravelmente. Este fato implica em competição acirrada por apoio financeiro, principalmente tendo em vista que os recursos provenientes dos órgãos de fomento à pesquisa, especialmente os governamentais, são cada vez mais limitados e insuficientes ${ }^{6}$.

Buscando excelência no ensino aliando-o a pesquisa, a EBMSP também possui dois tipos de Bolsa de Iniciação Científica (PIBIC), sendo a PIBIC-Bahiana com recursos próprios e a PIBIC-FABESB com financiamento do Estado.

Prótese, CTBMF e estomatologia foram as áreas preponderantes para relatos de caso em TCC. Já era esperado um relevante número de revisões de literatura nas áreas de Estomatologia e CTBMF devido ao fato de essas áreas muitas vezes lidarem com patologias de baixa prevalência e incidência, o que dificulta a realização de pesquisas, devido ao pequeno número de pacientes para amostra. Esse é um fator que dificulta a realização, mas, como mostrado nessa pesquisa, não é uma limitação intransponível, visto que estomatologia ficou em terceiro lugar nos trabalhos de pesquisa e CTBMF ficou em sexto.

Nas revisões de literatura, estomatologia, CTMBF e prótese foram mais uma vez as áreas de concentração mais escolhidas para realização de TCC.

A maioria dos trabalhos (293 $93,3 \%)$ não teve coorientador. O coorientador, presente em 21 (6,7\%) TCCs, pode ser um profissional da mesma área ou de área diferente. Nesse último caso, são trabalhos sobre temas que envolvem diferentes áreas da odontologia ou ciências da saúde.
Os trabalhos orientados e/ou coorientados por profissionais com doutorado somaram 186 (59,2\%), correspondendo a, praticamente, 6 de cada 10 TCCs. Nos trabalhos de revisão e relato de caso foi encontrada uma equivalência de TCCs orientados e/ou coorientados por doutores e não doutores. Já nos trabalhos de pesquisa, foi verificada uma desproporção. Quase três quartos dos trabalhos foram orientados e/ou coorientados por doutores. Esses dados mostram que a titulação de doutorado não foi um fator relevante para a confecção de revisões ou relatos de caso, mas foi, sim, importante fator na produção de pesquisas. É fato conhecido o papel que os doutores e estagiários pós-doutorais têm como componente essencial na base do trabalho de pesquisa ${ }^{9}$.

\section{CONCLUSÃO}

Os trabalhos de pesquisa foram os mais encontrados para desenvolvimento de TCCs, seguidos dos relatos de caso e revisões de literatura. Prótese, estomatologia e CTBMF estiveram entre as áreas de mais selecionadas pelos alunos, tanto em uma avaliação global, quanto quando subdividas por tipos de trabalho. A titulação de doutorado do orientador mostrou-se um relevante fator na produção de pesquisas.

\section{REFERÊNCIAS}

1. Amorim KPC, Alves MSCF, Germano RM. A construção do conhecimento na Odontologia: a produção científica em debate. Acta Cir Bras. 2005; 20(1):8-11.

2. Conselho Nacional de Educação/Câmara de Educação Superior (Brasil). Resolução $\mathrm{n}^{0}$. 3, de 19 fevereiro de 2002. Diretrizes Curriculares Nacionais do Curso de Graduação em Odontologia. Diário Oficial da União 4 mar 2002; Seção 1.

3. Silveira JLG. Diretrizes Curriculares Nacionais para os Cursos de Graduação 
em Odontologia: historicidade, legalidade e legitimidade. Pesq. Bras. Odontoped Clin Integr. 2004; 4(2):151-6.

4. Brasil. Ministério da Educação e Cultura [home page na internet]. Perguntas frequentes sobre Educação Superior [acesso em 4 nov 2012]. Disponível em: http://www.luizrosa.edu.br/fatec/faculda de/informativos/1\%203\%2010/document os/PERGUNTAS_RESPOSTAS.pdf .

5. Amorim KPC, Alves MSCF, Germano RM, Costa ICC. A construção do saber em Odontologia: a produção científica de três periódicos brasileiros de 1990 a 2004. Interface Comunic Saúde Educ. 2007; 11(21):9-23.

6. Cavalcanti AL, Melo TRNB, Barroso KMA, Souza FEC, Maia AMA, Silva ALO. Perfil da pesquisa científica em odontologia realizada no Brasil. Pesq. Bras. Odontoped Clin Integr. 2004;4(2):99-104.

7. Finkler M, Calvo MC, Caetano JC, Ramos FRS. Um novo olhar bioético sobre as pesquisas odontológicas brasileiras. Ciênc Saúde Colet. 2009; 14(4):1205-14.

8. Garbin CAS, Garbin AJI, Diniz DG. Panorama atual dos comitês de ética em pesquisa nas faculdades de medicina e odontologia brasileiras. Bioethikos. 2008; 2(2):197-203.

9. Guimarães R. Desafios da pós-graduação em saúde humana no Brasil. Rev Saúde Públ. 2011;45(1):1-13.

\section{ABSTRACT \\ Profile of Dental Academic Research from EBMSP}

The National Curricular Directives (DCN) for the Courses of Odontology, Resolution CNE/CES from March 2002 say that for the conclusion of the Graduate Course the student shall write a supervised final paper. In the Dental Course from the Bahian School of Medicine and Public Health (EBMSP), the Final Paper (FP) shall be written in the form of a scientific article, being possible to write an original article, a literature review or a case report. This study aimed to evaluate the profile of academic research in the Dental Course at EBMSP, through the analysis of all the FP that had presented in the period of 2007 ( $2^{\text {nd }}$ term) to 2012 ( $1^{\text {st }}$ term). The papers were classified in research, literature review, case report and regarding the area. Out of the 314 final papers, 114 (36.3\%) were researches, 109 (34.7\%) case reports and 91 (29\%) were literature reviews. Prosthesis, stomatology, surgery, periodontology and dentistry were the areas that had been chosen the most. The studies were guided or co-guided by a professor with doctorate in $59.2 \%$ (186) of the cases. Out of the research studies 88 (72\%) had the guidance of a doctor, but this number was reduced to 54 (49.5\%) in case reports and 45 (49.5\%) in reviews. It can be concluded that prosthesis, stomatology and surgery were among the most selected areas, in a global evaluation as well as when they were sub divided according to types of study. The adviser's title of doctor was a relevant factor in the production of researches.

Descriptors: Dental Research. Teaching. Bibliometrics.

Correspondência para:

Renato Magalhães Costa

e-mail: renatomagalhaesc@hotmail.com

Rua Antônio Pedro, 422/201

Centro 48903-660 Juazeiro/BA 